\title{
EXISTENCE AND REGULARITY OF THE DENSITY FOR THE SOLUTION TO SEMILINEAR DISSIPATIVE PARABOLIC SPDES
}

\author{
CARLO MARINELLI, EULALIA NUALART, AND LLUÍS QUER-SARDANYONS
}

\begin{abstract}
We prove existence and smoothness of the density of the solution to a nonlinear stochastic heat equation on $L^{2}(\mathcal{O})$ (evaluated at fixed points in time and space), where $\mathcal{O}$ is an open bounded domain in $\mathbb{R}^{d}$ with smooth boundary. The equation is driven by an additive Wiener noise and the nonlinear drift term is the superposition operator associated to a real function which is assumed to be (maximal) monotone, continuously differentiable, and growing not faster than a polynomial. The proof uses tools of the Malliavin calculus combined with methods coming from the theory of maximal monotone operators.
\end{abstract}

\section{INTRODUCTION}

Let $\mathcal{O} \subset \mathbb{R}^{d}$ be an open bounded domain, and consider the stochastic semilinear parabolic evolution equation on $L^{2}(\mathcal{O})$

$$
d u(t)-\Delta u(t) d t+f(u(t)) d t=\eta u(t) d t+B d W(t), \quad u(0)=u_{0},
$$

where $\Delta$ is the Dirichlet Laplacian on $L^{2}(\mathcal{O}), W$ is a standard cylindrical Wiener process on $L^{2}(\mathcal{O}), f: \mathbb{R} \rightarrow \mathbb{R}$ is continuous, increasing and of polynomial growth, $\eta$ is a positive number, $B$ is a deterministic bounded linear operator on $L^{2}(\mathcal{O})$, and $u_{0}$ is an $L^{2}(\mathcal{O})$ valued random variable (precise assumptions are given in the next section). As explained in Remark 2.1, the above equation covers the case where $f-\eta$ is replaced by $f+g$, where $f$ is as above and $g$ is a globally Lipschitz function.

Under regularity assumptions on the initial datum and the coefficients, one can prove, using monotonicity methods, that (1.1) admits a unique mild solution with continuous paths (see e.g. 4] and references therein). The purpose of this paper is to study the existence and regularity of the density (with respect to Lebesgue measure) of the random variable $u(t, x)$, with $(t, x) \in] 0, T] \times \mathcal{O}$. In particular, we show that a sufficient condition for the existence of the density is that $f$ is of class $C^{1}$ with polynomially bounded derivative. We do not claim that this condition is sharp (as a matter of fact, we conjecture that it is not, by far), but we do hope that our methods could be the starting point for further developments. The proof relies on techniques of the Malliavin calculus and on a priori estimates for solutions of approximating equations, obtained replacing the nonlinear drift term by its Yosida regularization. Furthermore, we show that if $f$ is of class $C^{m}$ with polynomially bounded derivatives, the density becomes smoother, as it is natural to expect. The proof of this still relies on a priori estimates, and requires a

Date: 21 February 2012.

2000 Mathematics Subject Classification. 60H07, 60H15.

Key words and phrases. Stochastic partial differential equation, existence and regularity of densities, Malliavin calculus. 
further regularization of the drift, as the Yosida approximation does not necessarily have bounded derivatives.

There is a large literature on problems of existence and regularity of densities for solutions to parabolic SPDEs with Lipschitz non-linearities by means of the Malliavin calculus (see [14, 16, 18, 19, 21 and references therein). On the other hand, much less attention has been dedicated to SPDEs with non-Lipschitz coefficients: the first article (relying on Malliavin calculus) we are aware of is the article [20, where a nonlinear one-dimensional stochastic heat equation on $[0,1]$ with polynomially growing drift and diffusion coefficients is considered. Using techniques of the Malliavin calculus, the authors prove, under quite general conditions, including a very weak non-degeneracy assumption on the diffusion coefficient, the absolute continuity of the law of the solution evaluated at fixed points in time and space. We note that existence and uniqueness of solution for the stochastic heat equation on $[0,1]$ driven by an additive noise with a measurable drift having polynomial growth has been proved in [10]. In [8], the absolute continuity for the law of the solution to a one-dimensional stochastic heat equation with Hölder continuous diffusion coefficient and linearly growing drift is proved with completely different methods. In particular, the proofs involve the Euler approximation and techniques of harmonic analysis.

As far as applications of Malliavin calculus to non-linear parabolic SPDEs is concerned, we should mention that the existence of the density for the stochastic CahnHilliard equation with non-Lipschitz coefficients has been investigated in [3. Furthermore, the existence and smoothness of the density for the solution to the stochastic Burgers equation with globally Lipschitz coefficients have been considered in [12, 23].

The paper is organized as follows. In Section 2, we provide the definition of mild solution to our equation (1.1), state a well-posedness result for this equation and summarize some properties of a regularized version of (1.1) in terms of the Yosida approximations. Section 3 is devoted to establish the random field counterpart (i.e. à la Walsh [22]) of our equation. In Section 4, we collect some auxiliary results needed for the main theorem's proof. These correspond to a version of the chain rule for Malliavin derivatives, some properties of time-dependent evolution operators and estimates for some regularizations of the drift coefficient. Eventually, in Section 5 , we state and prove the main result of the paper, Theorem [5.1. For this, we first deal with the Malliavin differentiability of the solution to our equation and, secondly, we study the invertibility of the corresponding Malliavin matrix.

Notation. We shall write $a \lesssim b$ to mean that there exists a constant $N$ such that $a \leq N b$. To emphasize that the constant $N$ depends on the parameters $p_{1}, \ldots, p_{m}$, we shall also write $a \lesssim_{1, \ldots, p_{m}} b$. We shall write sup to denote both the supremum and the essential supremum. For a Banach space $E$, we shall denote by $\mathbb{L}^{p}(E)$ the space of $E$-valued random variables $\xi$ such that $\mathbb{E}\|\xi\|_{E}^{p}<\infty$.

\section{WELL-POSEDNESS AND APPROXIMATION}

Let $\mathcal{O}$ be an open bounded domain in $\mathbb{R}^{d}$. $L^{p}$ spaces over the domain $\mathcal{O}$ will be denoted without explicitly mentioning the domain, e.g. $L^{2}$ stands for $L^{2}(\mathcal{O})$. The norm in $L^{p}$ will be denoted by $\|\cdot\|_{p}$, and the inner product of $L^{2}$ by $\langle\cdot, \cdot\rangle_{2}$, unless otherwise stated. 
On a given stochastic basis $\left(\Omega, \mathcal{F}, \mathbb{F}=\left(\mathcal{F}_{t}\right)_{0 \leq t \leq T}, \mathbb{P}\right)$, with $T$ a fixed positive number, let us be given the following semilinear SPDE on $L^{2}$ :

$$
d u(t)-\Delta u(t) d t+f(u(t)) d t=\eta u(t) d t+B d W(t), \quad u(0)=u_{0},
$$

where $\Delta$ is the Dirichlet Laplacian on $L^{2}, f: \mathbb{R} \rightarrow \mathbb{R}$ is continuous, increasing, and such that $|f(x)| \lesssim 1+|x|^{p}$ for some $p>0$ (we denote the evaluation operator associated to the function $f$ by the same symbol), $\eta$ is a positive number, $W$ is a cylindrical Wiener process on $L^{2}$ generating the filtration $\mathbb{F}, B: L^{2} \rightarrow L^{2}$ is a linear and bounded operator, and $u_{0}$ is an $\mathcal{F}_{0}$-measurable $L^{2}$-valued random variable such that $\mathbb{E}\left\|u_{0}\right\|_{2}^{2}<\infty$. All expressions involving random quantities are meant to hold $\mathbb{P}$-a.s. if not otherwise specified.

Remark 2.1. Instead of (2.1) one may equivalently consider an SPDE of the type

$$
d u(t)-\Delta u(t) d t+f(u(t)) d t=B d W(t), \quad u(0)=u_{0},
$$

where $f$ is quasi-monotone, i.e. such that $f+\eta$ is monotone for some $\eta>0$. In fact, we may write $f=(f+\eta)-\eta=\tilde{f}-\eta$, with $\tilde{f}$ monotone, thus obtaining (2.1) with $\tilde{f}$ replacing $f$. Furthermore, note that equations of the type

$$
d u(t)-\Delta u(t) d t+f(u(t)) d t+g(u(t)) d t=B d W(t), \quad u(0)=u_{0},
$$

with $f$ monotone (or quasi-monotone) and $g$ (globally) Lipschitz are just particular cases of (2.1). In fact, one has

$$
(f(x)+g(x)-f(y)-g(y))(x-y) \geq(g(x)-g(y))(x-y) \geq-\|g\|_{\dot{C}^{0,1}}|x-y|^{2},
$$

i.e. $f+g$ is quasi-monotone, since $f+g+\eta$ is monotone, with $\eta=\|g\|_{\dot{C}^{0,1}}$. (Here $\|\cdot\|_{\dot{C}^{0,1}}$ stands for the Lipschitz norm).

We shall work with the so-called mild solution, whose definition we recall. In the following we shall denote by $\Delta$ the realization of the Dirichlet Laplacian on different function spaces. The same convention we are going to use for the semigroup $S(t):=e^{t \Delta}$, $t \geq 0$, generated by $\Delta$.

Definition 2.2. An $L^{2}$-valued adapted process $u$ is a mild solution to equation (2.1) if $f(u) \in L^{1}\left([0, T] \rightarrow L^{2}\right)$ and it satisfies the integral equation

$$
u(t)+\int_{0}^{t} S(t-s)(f(u(s))-\eta u(s)) d s=S(t) u_{0}+\int_{0}^{t} S(t-s) B d W(s)
$$

for all $t \in[0, T]$.

As is well-known, in order for the stochastic integral in (2.2) to be a well-defined (Gaussian) $L^{2}$-valued random variable it is necessary (and sufficient) to assume that

$$
\int_{0}^{t} \operatorname{Tr}\left(S(s) B B^{*} S(s)^{*}\right) d s<\infty \quad \forall t \in[0, T] .
$$

Let us also recall that this condition is weaker than requiring $Q:=B B^{*}$ to be traceclass (cf. e.g. [5, Ch. 2]). We shall actually work under the following stronger standing assumption, which guarantees, as we are going to recall, that (2.1) admits a unique mild solution with paths in a space of continuous functions. 
Hypothesis 2.3. It holds

$$
\mathbb{E} \sup _{t \leq T}\left\|\int_{0}^{t} S(t-s) B d W(s)\right\|_{C(\overline{\mathcal{O}})}^{q}<\infty \quad \forall q \geq 1 .
$$

Conditions on $B$ and $\mathcal{O}$ implying that this hypothesis is fulfilled are extensively discussed in the literature (cf. e.g. [4, Ch. 6] and [5, Ch. 2]).

In order to state the well-posedness and approximation results we need, let us fix some further notation: we denote by $\mathbb{C}_{q}, 1 \leq q<\infty$, the space of adapted processes $u$ with values in $C(\overline{\mathcal{O}})$ such that

$$
\|u\|_{\mathbb{C}_{q}}:=\left(\mathbb{E} \sup _{t \leq T}\|u(t)\|_{C(\overline{\mathcal{O}})}^{q}\right)^{1 / q}<\infty .
$$

The following global well-posedness result holds true (see e.g. 6, Thm. 7.13 and Rmk. 11.23], as well as [4, Prop. 6.2.2], for a proof).

Theorem 2.4. Let $q \geq 1$ and assume that $u_{0} \in \mathbb{L}^{q}(C(\overline{\mathcal{O}}))$. Then equation (2.1) admits a unique mild solution $u \in \mathbb{C}_{q}$.

Let us now introduce the regularized SPDE

$$
d u_{\lambda}(t)-\Delta u_{\lambda}(t) d t+f_{\lambda}\left(u_{\lambda}(t)\right) d t=\eta u_{\lambda}(t) d t+B d W(t), \quad u(0)=u_{0},
$$

where $f_{\lambda}, \lambda>0$, stands for the Yosida approximation of $f$, i.e.

$$
f_{\lambda}(x):=\frac{1}{\lambda}\left(x-J_{\lambda}(x)\right), \quad J_{\lambda}(x):=(I+\lambda f)^{-1}(x) .
$$

Recall that $f_{\lambda}$ is increasing, Lipschitz continuous with Lipschitz constant bounded by $1 / \lambda$, and $f_{\lambda} \rightarrow f$ pointwise as $\lambda \rightarrow 0$. Moreover, $J_{\lambda}$ is a contraction and $f_{\lambda}=f \circ J_{\lambda}$ (see e.g. 2] for a detailed discussion of the Yosida approximation). It is then clear that Theorem 2.4 applies also to (2.4), yielding the existence and uniqueness of a mild solution $u_{\lambda} \in \mathbb{C}_{q}$. Furthermore, the following convergence result holds true (see [4, Prop. 6.2.5]).

Proposition 2.5. Let $q \geq 1$ and assume that $u_{0} \in \mathbb{L}^{q}(C(\overline{\mathcal{O}}))$. Then there exists a constant $N$, independent of $\lambda$, such that

$$
\mathbb{E} \sup _{t \leq T}\left\|u_{\lambda}(t)\right\|_{C(\overline{\mathcal{O}})}^{q}<N
$$

Moreover, one has

$$
\lim _{\lambda \rightarrow 0} \mathbb{E} \sup _{t \leq T}\left\|u_{\lambda}(t)-u(t)\right\|_{C(\overline{\mathcal{O}})}^{q}=0,
$$

where $u \in \mathbb{C}_{q}$ denotes the (unique) mild solution to equation (2.1). In particular, one has, for any $(t, x) \in[0, T] \times \overline{\mathcal{O}}$,

$$
\lim _{\lambda \rightarrow 0} \mathbb{E}\left|u_{\lambda}(t, x)-u(t, x)\right|^{q}=0 .
$$




\section{An Alternative expression For the SPDE (2.1)}

The pathwise continuity in $t$ and $x$ of the solution to equation (2.2) guaranteed by Theorem 2.4 allows us to pass to the random field formulation of (2.2), i.e. as in 22], in a sense made precise in Proposition 3.1 below.

It is classical that $S(t)$ is a kernel operator for all $t>0$, i.e. there exists a function ] $0, T] \times \mathcal{O} \times \mathcal{O} \ni(t, x, y) \mapsto G_{t}(x, y)$, with $G_{t}(\cdot, \cdot) \in L^{\infty}(\mathcal{O} \times \mathcal{O})$ for all $\left.\left.t \in\right] 0, T\right]$, such that, for any $0<t \leq T$,

$$
S(t) \phi=\int_{\mathcal{O}} G_{t}(\cdot, y) \phi(y) d y
$$

and

$$
\|S(t)\|_{1 \rightarrow \infty}=\left\|G_{t}(\cdot, \cdot)\right\|_{L^{\infty}(\mathcal{O} \times \mathcal{O})},
$$

where $\|\cdot\|_{1 \rightarrow \infty}$ stands for the $L^{1} \rightarrow L^{\infty}$ operator norm. Since the semigroup $S$ is contracting in $L^{\infty}$ i.e. $\|S(t) f\|_{L^{\infty}} \leq\|f\|_{L^{\infty}}$ for all $t>0$, and it holds

$$
G_{t}(x, \mathcal{O})=\int_{\mathcal{O}} G_{t}(x, y) d y=\left[S(t) 1_{\mathcal{O}}\right](x),
$$

one has

$$
\sup _{x \in \mathcal{O}} G_{t}(x, \mathcal{O})=\left\|S(t) 1_{\mathcal{O}}\right\|_{L^{\infty}} \leq 1 .
$$

We have the following result, where we use the terminology introduced in [22] for stochastic integrals.

Proposition 3.1. For $q \geq 1$, let $u_{0} \in \mathbb{L}^{q}(C(\mathcal{O}))$ and denote the unique mild solution in $\mathbb{C}_{q}$ to equation (2.2) by $u$. Setting $u(t, x):=[u(t)](x)$, one has, for any $\left.\left.(t, x) \in\right] 0, T\right] \times \mathcal{O}$,

$$
\begin{aligned}
u(t, x)= & \int_{\mathcal{O}} G_{t}(x, y) u_{0}(y) d y+\int_{0}^{t} \int_{\mathcal{O}} G_{t-s}(x, y)(\eta u(s, y)-f(u(s, y))) d y d s \\
& +\int_{0}^{t} \int_{\mathcal{O}} G_{t-s}(x, y) \bar{W}(d s, d y),
\end{aligned}
$$

where $\bar{W}$ stands for a martingale measure with covariance operator $Q=B B^{*}$. Moreover,

$$
\left.\mathbb{E} \sup _{(t, x) \in \mathcal{O}_{T}}|u(t, x)|^{q}\right)<\infty,
$$

where $\mathcal{O}_{T}:=[0, T] \times \mathcal{O}$.

Proof. It is readily seen that we only have to prove that, for any $t \in] 0, T]$,

$$
\int_{0}^{t} S(t-s) B d W(s)=\int_{0}^{t} \int_{\mathcal{O}} G_{t-s}(\cdot, y) \bar{W}(d s, d y)
$$

as $L^{2}$-valued random variables. Let us assume (without loss of generality) that, formally,

$$
W(t)=\sum_{k \in \mathbb{N}} e^{k} w_{k}(t)
$$


where $\left\{e^{k}\right\}_{k \in \mathbb{N}}$ is a basis of $L^{2}$ and $\left\{w_{k}\right\}_{k \in \mathbb{N}}$ is a family of independent standard onedimensional Brownian motions. Then we have, by the integral representation of $S(\cdot)$,

$$
\begin{aligned}
\int_{0}^{t} S(t-s) B d W(s) & =\sum_{k \in \mathbb{N}} \int_{0}^{t} S(t-s) B e^{k} d w_{k}(s) \\
& =\sum_{k \in \mathbb{N}} \int_{0}^{t} \int_{\mathcal{O}} G_{t-s}(\cdot, y)\left[B e^{k}\right](y) d y d w_{k}(s),
\end{aligned}
$$

where the series of ordinary Itô integrals are no longer formal by virtue of (2.3).

As far as the stochastic integral on the right-hand side of (3.3) is concerned, we notice (see e.g. [7]) that it may be understood as a stochastic integral with respect to a cylindrical $Q$-Wiener process $\left\{\bar{W}_{h}(t), h \in L^{2}, t \geq 0\right\}$ in the sense of e.g. [15]. Namely, by definition, the latter is a centered Gaussian family of random variables such that, for any $h \in L^{2}$, the process $\left\{\bar{W}_{h}(t), t \geq 0\right\}$ is a Brownian motion with variance $t\langle Q h, h\rangle$ and, for all $s, t \geq 0$ and $h, g \in L^{2}$,

$$
\mathbb{E}\left(\bar{W}_{h}(s) \bar{W}_{g}(t)\right)=(s \wedge t)\langle Q h, g\rangle_{2} .
$$

By an innocuous abuse of notation, this cylindrical $Q$-Wiener process will be also denoted by $\bar{W}$. Let us introduce the Hilbert space $L_{Q}^{2}$, which we define as the completion of $L^{2}$ with respect to $\langle h, g\rangle_{L_{Q}^{2}}:=\langle Q h, g\rangle_{2}$. Then one can define the (real-valued) stochastic integral with respect to $\bar{W}$ of any $L_{Q}^{2}$-valued square integrable process as follows: let $\left\{\bar{e}^{k}\right\}_{k \in \mathbb{N}}$ be a basis of $L_{Q}^{2}$ and $X \in L^{2}\left(\Omega \times[0, T] \rightarrow L_{Q}^{2}\right)$ (see e.g. [7, Sec. 2]), and set

$$
\int_{0}^{T} \int_{\mathcal{O}} X(s, y) \bar{W}(d s, d y):=\sum_{k \in \mathbb{N}} \int_{0}^{t}\left\langle X(s), \bar{e}^{k}\right\rangle_{L_{Q}^{2}} d \bar{W}_{\bar{e}_{k}}(s) .
$$

Moreover, the $L^{2}$-valued cylindrical Wiener process $W$ of (3.4) determines a cylindrical $Q$-Wiener process $\bar{W}$, with $Q=B B^{*}$, as follows: for any $t \geq 0$ and $h \in L^{2}$, set

$$
\bar{W}_{h}(t):=\sum_{k \in \mathbb{N}} \int_{0}^{t}\left\langle B e^{k}, h\right\rangle_{2} d w_{k}(s) .
$$

It is immediate that $\bar{W}_{h}(t)$ is a centered Gaussian random variable and

$$
\mathbb{E}\left(\sum_{k} \int_{0}^{t}\left\langle B e^{k}, h\right\rangle_{2} d w_{k}(s)\right)^{2}=t \sum_{k}\left\langle e^{k}, B^{*} h\right\rangle_{2}^{2}=t\left\|B^{*} h\right\|_{2}^{2}=t\left\langle B B^{*} h, h\right\rangle_{2}=t\langle Q h, h\rangle_{2} .
$$

In a completely similar way one verifies the covariance condition (3.5).

In order to prove (3.3), one just needs to take $\bar{e}^{k}:=Q^{-1 / 2} e^{k}$, where $Q^{-1 / 2}$ denotes the pseudo-inverse of $Q^{1 / 2}$, whence one easily verifies that $\bar{W}_{\bar{e}^{k}}(s)=w_{k}(s)$ and

$$
\int_{\mathcal{O}} G_{t-s}(\cdot, y)\left[B e^{k}\right](y) d y=\int_{\mathcal{O}} G_{t-s}(\cdot, y)\left[Q \bar{e}^{k}\right](y) d y .
$$

We have thus proved the identities

$$
\int_{0}^{t} S(t-s) B d W(s)=\sum_{k} \int_{0}^{t} \int_{\mathcal{O}} G_{t-s}(\cdot, y)\left[B e^{k}\right](y) d w_{k}(s)=\int_{0}^{t} \int_{\mathcal{O}} G_{t-s}(\cdot, y) \bar{W}(d s, d y) .
$$

Finally, the estimate (3.2) is an immediate consequence of Theorem 2.4. 


\section{Auxiliary Results}

We collect here some tools that we shall need in the next section. In particular, we give a version of the chain rule for the Malliavin derivative, where a (Malliavin) differentiable random variable is composed with an increasing function of polynomial growth. The result, also without the monotonicity assumption, is certainly well-known to experts (cf. e.g. [13, p.36]), but we include a proof for completeness and because most standard references contain only a proof for functions of class $C_{b}^{1}$ (cf. e.g. [17, Prop. 1.2.3]). Moreover, we prove some estimates for evolution operators generated by time-dependent bounded perturbations of the Laplacian and for regularizations via Yosida approximations as well as via mollification.

As usual, a function $\phi: \mathbb{R} \rightarrow \mathbb{R}$ is said to be of polynomial growth if there exists $p \in \mathbb{N}$ such that $|\phi(x)| \lesssim 1+|x|^{p}$ for all $x \in \mathbb{R}$. We shall denote by $C_{\mathrm{pol}}^{k}(\mathbb{R})$ the space of functions $\phi \in C^{k}(\mathbb{R})$ such that $\phi, \phi^{\prime}, \ldots, \phi^{(k)}$ are of polynomial growth. It is not difficult to see that, by the fundamental theorem of calculus, one can equivalently say that $C_{\mathrm{pol}}^{k}(\mathbb{R})$ is the space of functions $\phi \in C^{k}(\mathbb{R})$ such that $\phi^{(k)}$ is of polynomial growth, and also that it is the space of functions $\phi \in C^{k}(\mathbb{R})$ for which there exists $p \in \mathbb{N}$ such that

$$
|\phi(x)|+\left|\phi^{\prime}(x)\right|+\cdots+\left|\phi^{(k)}(x)\right| \lesssim 1+|x|^{p} .
$$

4.1. Malliavin calculus. We shall repeatedly use the following strong-weak closability property of the Malliavin derivative (cf. e.g. [18, p. 78]). We use standard notation and terminology (see e.g. [17]).

Lemma 4.1. Let $k \in \mathbb{N}, p \in] 1, \infty\left[\right.$. Assume that $\lim _{n \rightarrow \infty} X_{n}=X$ in $L^{p}(\Omega)$ and $\sup _{n \in \mathbb{N}}\left\|X_{n}\right\|_{\mathbb{D}^{k, p}}<\infty$. Then $X \in \mathbb{D}^{k, p}$ and $X_{n} \rightarrow X$ weakly in $\mathbb{D}^{k, p}$.

We shall denote the Gaussian Hilbert space "supporting" the Malliavin calculus by $H$. Moreover, we shall say that $X \in \mathbb{D}^{k, \infty}$ if $X \in \mathbb{D}^{k, p}$ for all $p>1$.

Lemma 4.2. Let $X \in \mathbb{D}^{1, \infty}, f \in C_{\text {pol }}^{1}(\mathbb{R})$ and increasing. Then $f(X) \in \mathbb{D}^{1, \infty}$ and $D f(X)=f^{\prime}(X) D X$.

Proof. Assume, without loss of generality, that $|f(x)|+\left|f^{\prime}(x)\right| \lesssim 1+|x|^{p}$, with $p \in \mathbb{N}$. Let $f_{\lambda}, \lambda>0$, denote the Yosida approximation of $f$. Since $f_{\lambda} \rightarrow f$ pointwise and $f$ is continuous, one has $f_{\lambda}(X) \rightarrow f(X) \mathbb{P}$-a.s. as $\lambda \rightarrow 0$. Moreover, recalling that $\left|f_{\lambda}\right| \leq|f|$, hence that, for any $q \geq 1$,

$$
\left|f_{\lambda}(x)-f(x)\right|^{q} \leq 2^{q}|f(x)|^{q} \lesssim 1+|x|^{q p},
$$

and $\mathbb{E}|X|^{q p}<\infty$, the dominated convergence theorem yields

$$
\lim _{\lambda \rightarrow 0} \mathbb{E}\left|f_{\lambda}(X)-f(X)\right|^{q}=0 \quad \forall q \geq 1 .
$$

Appealing to the inverse function theorem, it is easy to show that $f \in C^{1}$ implies $f_{\lambda}^{\prime} \in C_{b}$, and

$$
J_{\lambda}^{\prime}(x)=\frac{1}{1+\lambda f^{\prime}\left(J_{\lambda}(x)\right)}, \quad f_{\lambda}^{\prime}(x)=\frac{f^{\prime}\left(J_{\lambda}(x)\right)}{1+\lambda f^{\prime}\left(J_{\lambda}(x)\right)} .
$$


In particular, since $f_{\lambda}$ is Lipschitz continuous, one has $f_{\lambda} \in C_{b}^{1}$, so that the "classical" chain rule (see e.g. [17, Prop. 1.2.3]) implies that $f_{\lambda}(X) \in \mathbb{D}^{1, \infty}$ and $D f_{\lambda}(X)=$ $f_{\lambda}^{\prime}(X) D X$. Let us check now that, for any $q \geq 1$, it holds

$$
\sup _{\lambda>0} \mathbb{E}\left\|D f_{\lambda}(X)\right\|_{H}^{q}<\infty .
$$

By the above expression for $f_{\lambda}^{\prime}$ and $f^{\prime} \geq 0$ it immediately follows that

$$
\left|f_{\lambda}^{\prime}(x)\right| \leq\left|f^{\prime}\left(J_{\lambda}(x)\right)\right| \lesssim 1+\left|J_{\lambda}(x)\right|^{p} \leq 1+|x|^{p},
$$

hence, by Cauchy-Schwarz' inequality,

$$
\mathbb{E}\left\|D f_{\lambda}(X)\right\|_{H}^{q} \leq\left(\mathbb{E}\left|f_{\lambda}^{\prime}(X)\right|^{2 q}\right)^{1 / 2}\left(\mathbb{E}\|D X\|_{H}^{2 q}\right)^{1 / 2} \lesssim\left(1+\mathbb{E}|X|^{2 p q}\right)^{1 / 2}\left(\mathbb{E}\|D X\|_{H}^{2 q}\right)^{1 / 2} .
$$

The right-hand side is finite and independent of $\lambda$, thus (4.2) is proved. In particular, (4.1) and (4.2) imply that $f(X) \in \mathbb{D}^{1, \infty}$ and $D f_{\lambda}(X)$ converges to $D f(X)$ weakly in $\mathbb{L}^{q}(H)$ as $\lambda \rightarrow 0$, for all $q \geq 1$. Since the weak limit is unique, in order to prove that $D f(X)=f^{\prime}(X) D X$, it suffices to show that

$$
\lim _{\lambda \rightarrow 0} \mathbb{E}\left\|D f_{\lambda}(X)-f^{\prime}(X) D X\right\|_{H}^{q}=0 .
$$

Observe that

$$
\begin{aligned}
\mathbb{E}\left\|D f_{\lambda}(X)-f^{\prime}(X) D X\right\|_{H}^{q} & =\mathbb{E}\left(\left|f_{\lambda}^{\prime}(X)-f^{\prime}(X)\right|^{q}\|D X\|_{H}^{q}\right) \\
& \leq\left(\mathbb{E}\left|f_{\lambda}^{\prime}(X)-f^{\prime}(X)\right|^{2 q}\right)^{1 / 2}\left(\mathbb{E}\|D X\|_{H}^{2 q}\right)^{1 / 2},
\end{aligned}
$$

and that, by the expression of $f_{\lambda}^{\prime}$, one has $f_{\lambda}^{\prime} \rightarrow f^{\prime}$, hence $f_{\lambda}^{\prime}(X) \rightarrow f^{\prime}(X) \mathbb{P}$-a.s. as $\lambda \rightarrow 0$. Moreover, taking into account that

$$
\left|f_{\lambda}^{\prime}(x)-f^{\prime}(x)\right|=\left|\frac{f^{\prime}\left(J_{\lambda}(x)\right)}{1+\lambda f^{\prime}\left(J_{\lambda}(x)\right)}-f^{\prime}(x)\right| \leq\left|f^{\prime}\left(J_{\lambda}(x)\right)\right|+\left|f^{\prime}(x)\right| \lesssim 1+|x|^{p},
$$

one also has

$$
\left|f_{\lambda}^{\prime}(X)-f^{\prime}(X)\right|^{2 q} \lesssim 1+|X|^{2 p q}
$$

hence, by the dominated convergence theorem, $f_{\lambda}^{\prime}(X) \rightarrow f^{\prime}(X)$ in $\mathbb{L}^{2 q}$ as $\lambda \rightarrow 0$, and the proof is finished.

4.2. Time-dependent evolution operators. Using the notation introduced in Section 2, let $F:[0, T] \rightarrow L_{+}^{\infty}$, and consider the following linear evolution equation on $L^{2}$ :

$$
d y(t)-\Delta y(t)+F(t) y(t)=0, \quad y(s)=y_{0} \in L^{2}, \quad 0 \leq s \leq t \leq T .
$$

Here $L_{+}^{p}, p \in[1, \infty]$, denotes the set of (equivalence classes of) functions $\phi \in L^{p}$ such that $\phi \geq 0$ a.e.. The evolution operator $U(t, s)$ is then defined by $y(t)=: U(t, s) y_{0}$.

Proposition 4.3. For any $0 \leq s<t \leq T$, the following properties hold true:

(i) $U(t, s)$ is positivity preserving, i.e. $y_{0} \geq 0$ implies $U(t, s) y_{0} \geq 0$;

(ii) $U(t, s) \leq S(t-s)$, i.e. $y_{0} \geq 0$ implies $U(t, s) y_{0} \leq S(t-s) y_{0}$;

(iii) $U(t, s)$ is ultracontractive, i.e. its $L^{1} \rightarrow L^{\infty}$ norm is finite. 
(iv) $U(t, s)$ is a kernel operator, i.e. there exists a function $k:[0, T]^{2} \times \mathcal{O}^{2} \rightarrow \mathbb{R}_{+}$ such that

$$
[U(t, s) \phi](x)=\int_{\mathcal{O}} k(t, s ; x, y) \phi(y) d y .
$$

Proof. (i) Let $y_{0} \geq 0$ and $y(\cdot)$ be a strong solution (without loss of generality) of (4.3), where we assume, for simplicity, $s=0$. Taking the scalar product with $y^{-}$and integrating with respect to time, we obtain, denoting the $L^{2}$ norm by $\|\cdot\|$,

$$
\frac{1}{2}\left\|y^{-}(t)\right\|^{2}+\int_{0}^{t}\left\|\nabla y^{-}(r)\right\|^{2} d r+\int_{0}^{t}\left\langle F(r) y^{-}(r), y^{-}(r)\right\rangle d r=\left\|y_{0}^{-}\right\|^{2},
$$

hence $\left\|y^{-}(t)\right\|^{2} \leq 2\left\|y_{0}^{-}\right\|^{2}=0$, i.e. $y(t) \geq 0$ for all $t \in[0, T]$. (ii) Let $z$ be the solution to $z^{\prime}-\Delta z=0, z(0)=y_{0}$. Then one has

$$
\frac{d}{d t}(y-z)(t)-\Delta(y-z)(t)+F(t) y(t)=0, \quad(y-z)(0)=0,
$$

hence, taking the scalar product with $(y-z)^{+}$and integrating,

$$
\left\|(y(t)-z(t))^{+}\right\|^{2}+\int_{0}^{t}\left\|\nabla(y(r)-z(r))^{+}\right\|^{2} d r+\int_{0}^{t}\left\langle F(r) y(r),(y(r)-z(r))^{+}\right\rangle d s=0,
$$

which yields, recalling that, by (i), $y(r) \geq 0$ for all $r, y(t) \leq z(t)$ for all $t$. (iii) Note that, by (i), one has

$$
\begin{aligned}
\left|U(t, s) y_{0}\right|=\left|U(t, s) y_{0}^{+}-U(t, s) y_{0}^{-}\right| & \leq\left|U(t, s) y_{0}^{+}\right|+\left|U(t, s) y_{0}^{-}\right| \\
& =U(t, s) y_{0}^{+}+U(t, s) y_{0}^{-}=U(t, s)\left|y_{0}\right|,
\end{aligned}
$$

therefore

$$
\left|U(t, s) y_{0}\right| \leq U(t, s)\left|y_{0}\right| \leq S(t-s)\left|y_{0}\right| \in L^{\infty},
$$

which immediately implies $U(t, s) y_{0} \in L^{\infty}$. (iv) is a direct consequence of (iii), thanks to a classical criterion of Dunford and Pettis (see e.g. [11, Ch. XI, §1]).

4.3. Regularizations. In the next Lemma we use the notation introduced immediately after Theorem 2.4 .

Lemma 4.4. Let $f \in C_{\mathrm{pol}}^{m}(\mathbb{R})$ be an increasing function such that $\left|f^{(n)}(x)\right| \lesssim 1+|x|^{p}$ for all $n=0,1, \ldots, m$. Then the following properties hold:

(i) $f_{\lambda}$ and $J_{\lambda}$ belong to $C^{m}(\mathbb{R})$;

(ii) for $\lambda \leq 1$, there exists $q \in \mathbb{N}$, independent of $\lambda$, such that $\left|f_{\lambda}^{(n)}(x)\right| \lesssim 1+|x|^{q}$ for all $n=0,1, \ldots, m$.

(iii) $f_{\lambda}^{(n)}$ converges pointwise to $f^{(n)}$ as $\lambda \rightarrow 0$.

Proof. Since $J_{\lambda}=(I+\lambda f)^{-1}$, and $f^{\prime} \geq 0$, the inverse function theorem implies that, if $f$ is of class $C^{m}$, then also $J_{\lambda}$ is of class $C^{m}$. Moreover, the identity $\lambda f_{\lambda}=I-J_{\lambda}$ implies that also $f_{\lambda}$ is of class $C^{m}$, hence (i) is proved.

(ii) The polynomial growth of $f_{\lambda}$ is obvious by the inequality $\left|f_{\lambda}\right| \leq|f|$. Moreover, recalling that $f_{\lambda}=f \circ J_{\lambda}$, that $J_{\lambda}$ is of class $C^{m}$ and is a contraction, we have

$$
\left|f_{\lambda}^{\prime}(x)\right|=\left|f^{\prime}\left(J_{\lambda}(x)\right)\right|\left|J_{\lambda}^{\prime}(x)\right| \leq\left|f^{\prime}\left(J_{\lambda}(x)\right)\right| \lesssim 1+\left|J_{\lambda}(x)\right|^{p} \leq 1+|x|^{p} .
$$


Taking into account that $\lambda f_{\lambda}^{\prime \prime}=J_{\lambda}^{\prime \prime}$ and that

$$
f_{\lambda}^{\prime \prime}=f^{\prime \prime}\left(J_{\lambda}\right)\left(J_{\lambda}^{\prime}\right)^{2}+f^{\prime}\left(J_{\lambda}\right) J_{\lambda}^{\prime \prime},
$$

one gets $\left(1+\lambda f^{\prime}\left(J_{\lambda}\right)\right) f_{\lambda}^{\prime \prime}=f^{\prime \prime}\left(J_{\lambda}\right)\left(J_{\lambda}^{\prime}\right)^{2}$, which implies

$$
\left|f_{\lambda}^{\prime \prime}(x)\right| \leq\left|f^{\prime \prime}\left(J_{\lambda}(x)\right)\right| \lesssim 1+\left|J_{\lambda}(x)\right|^{p} \leq 1+|x|^{p} .
$$

Unfortunately it does not seem possible to extend such elementary arguments to obtain the polynomial growth of $f_{\lambda}^{(n)}$. We can nonetheless argue as follows: by Arbogast's formula [ (see e.g. 9]) one has

$$
f_{\lambda}^{(n)}=\sum \frac{n !}{b_{1} ! b_{2} ! \cdots b_{n} !} f^{(k)}\left(J_{\lambda}\right)\left(\frac{J_{\lambda}^{\prime}}{1 !}\right)^{b_{1}}\left(\frac{J_{\lambda}^{\prime \prime}}{2 !}\right)^{b_{2}} \cdots\left(\frac{J^{(n)}}{n !}\right)^{b_{n}}
$$

where the sum is taken over all possible combinations of $\left\{b_{1}, b_{2}, \ldots, b_{n}\right\} \subset \mathbb{N} \cup\{0\}$ such that

$$
b_{1}+2 b_{2}+\cdots+n b_{n}=n \quad \text { and } \quad b_{1}+b_{2}+\cdots+b_{n}=k .
$$

In particular, note that there is only one possible term of the series containing $f^{\prime}\left(J_{k}\right)$, precisely the one corresponding to $b_{1}=b_{2}=\cdots=b_{n-1}=0, b_{n}=1$, that is $f^{\prime}\left(J_{\lambda}\right) J_{\lambda}^{(n)}$. Similarly, there is only one possible term containing $f^{(n)}\left(J_{\lambda}\right)$, precisely the one corresponding to $b_{1}=n, b_{2}=b_{3}=\cdots=b_{n}=0$, that is $f^{(n)}\left(J_{\lambda}\right)\left(J_{\lambda}^{\prime}\right)^{n}$. Recalling that $\lambda f_{\lambda}^{(n)}=-J_{\lambda}^{(n)}$, we have

$$
\left(1+\lambda f^{\prime}\left(J_{\lambda}\right)\right) f_{\lambda}^{(n)}=f^{(n)}\left(J_{\lambda}\right)\left(J_{\lambda}^{\prime}\right)^{n}+S_{n-1},
$$

hence

$$
\left|f_{\lambda}^{(n)}\right| \leq\left|f^{(n)}\left(J_{\lambda}\right)\right|+\left|S_{n-1}\right|
$$

where $S_{n-1}$ is a finite sum of terms involving only $f^{(k)}\left(J_{\lambda}\right)$ and powers of $J_{\lambda}^{(k)}$, for $k=1, \ldots, n-1$. Using once again that $\lambda f_{\lambda}^{(k)}=-J_{\lambda}^{(k)}$, we conclude that $S_{n-1}$ is a finite sum of terms involving only $f^{(k)}\left(J_{\lambda}\right)$ and powers of $\lambda f_{\lambda}^{(k)}$, for $k=1, \ldots, n-1$. Taking $\lambda \leq 1$, since $\left|f^{(k)}\left(J_{\lambda}(x)\right)\right| \lesssim 1+|x|^{p}$ for all $k=1, \ldots, n$, recalling that $f_{\lambda}^{\prime \prime}$ satisfies (4.4), we obtain that there exists $q_{3} \in \mathbb{N}$ such that $\left|f_{\lambda}^{(3)}(x)\right| \lesssim 1+|x|^{q_{3}}$. By iteration one ends up with $\left|f_{\lambda}^{(k)}(x)\right| \lesssim 1+|x|^{q_{k}}$ for each $k=0,1, \ldots, m$. Since $m$ is finite, this implies the claim. (iii) It is known that $f_{\lambda} \rightarrow f$ pointwise as $\lambda \rightarrow 0$. That $f_{\lambda}^{\prime} \rightarrow f^{\prime}$ pointwise as $\lambda \rightarrow 0$ has been proved in Lemma 4.2. Passing to the limit (pointwise) as $\lambda \rightarrow 0$ in (4.5) we obtain

$$
\lim _{\lambda \rightarrow 0} f_{\lambda}^{(n)}=f^{(n)}+\lim _{\lambda \rightarrow 0} S_{n-1},
$$

where we have used that $J_{\lambda} \rightarrow I$ and

$$
\lim _{\lambda \rightarrow 0} J_{\lambda}^{\prime}(x)=\lim _{\lambda \rightarrow 0} \frac{1}{1+\lambda f^{\prime}\left(J_{\lambda}(x)\right)}=1 .
$$

\footnotetext{
${ }^{1}$ This formula is better known as Faà di Bruno's formula. The latter attribution, however, seems to be historically incorrect.
} 
The claim is proved if we show that $S_{n-1} \rightarrow 0$ pointwise as $\lambda \rightarrow 0$. For $n=2$ one has $S_{1}=0$, hence the claim holds. For $n \geq 3$, we observe that each term in $S_{n-1}$ is of the form

$$
c f^{(i)}\left(J_{\lambda}\right)\left(J_{\lambda}^{\prime}\right)^{h_{1}}\left(J_{\lambda}^{\prime \prime}\right)^{h_{2}} \cdots\left(J_{\lambda}^{(n-1)}\right)^{h_{n-1}},
$$

where $c$ is a positive number, $1 \leq i, h_{1} \leq n-1$, and $h_{2}, h_{3}, \ldots, h_{n-1}$ are nonnegative integers with at least one of them, say $h_{s}$, greater than 1 . Let $2 \leq \sigma:=h_{s}$. Recall that

$$
J_{\lambda}^{(\sigma)}=-\lambda f_{\lambda}^{(\sigma)},
$$

therefore the generic term of $S_{n-1}$, hence $S_{n-1}$ itself, converges to zero as $\lambda \rightarrow 0$.

Let us introduce mollifiers in the following (standard) way: for $\zeta \in C^{\infty}(\mathbb{R})$ positive, with support contained in $[-1,1]$ and $\int_{\mathbb{R}} \zeta=1$, set, for any $\beta>0, \zeta_{\beta}(x):=\beta^{-1} \zeta(x / \beta)$.

Lemma 4.5. Let $f: \mathbb{R} \rightarrow \mathbb{R}$ be such that $|f(x)| \lesssim 1+|x|^{p}$, and $f_{\beta}=f * \zeta_{\beta}, \beta \leq 1$. Then one has

$$
\left|f_{\beta}(x)\right| \leq N\left(1+|x|^{p}\right) \quad \forall x \in \mathbb{R}
$$

where the constant $N$ does not depend on $\beta$.

Proof. Assume that $|f(x)| \leq N_{1}\left(1+|x|^{p}\right)$. By the triangle inequality and the properties of $\zeta$ one has

$$
\begin{aligned}
\left|f_{\beta}(x)\right| & \leq \int_{\mathbb{R}}|f(x-y)| \zeta_{\beta}(y) d y \leq N_{1} \int_{\mathbb{R}}\left(1+|x-y|^{p}\right) \zeta_{\beta}(y) d y \\
& \leq N_{1}\left(1+|x|^{p}\right)+N_{1} \int_{\mathbb{R}}|y|^{p} \zeta_{\beta}(y) d y \\
& =N_{1}\left(1+|x|^{p}\right)+N_{1} \beta^{p} \int_{\mathbb{R}}|y|^{p} \zeta(y) d y \\
& \leq N_{1}\left(2+|x|^{p}\right) .
\end{aligned}
$$

\section{Existence AND SMOOThness of The DENSity}

In this section, we provide sufficient conditions on the data of the problem implying that the law of $u(t, x)$ is absolutely continuous with respect to the Lebesgue measure and that the corresponding density is a differentiable function. More precisely, we will prove the following result.

Theorem 5.1. Assume that $u_{0} \in C(\overline{\mathcal{O}})$ and let $u \in \bigcap_{q \in \mathbb{N}} \mathbb{C}_{q}$ be the mild solution to (2.1). Furthermore, assume that there exists $\gamma \in(0,2)$ such that, for all $x \in \mathcal{O}$, there exists a constant $c_{x}>0$ such that, for any $t \in(0,1)$,

$$
g(x, t):=\int_{0}^{t}\left\|G_{s}(x, \cdot)\right\|_{L_{Q}^{2}}^{2} d s \geq c_{x} t^{\gamma} .
$$

(a) If $f \in C_{\text {pol }}^{1}(\mathbb{R})$ is increasing, then for any $\left.\left.(t, x) \in\right] 0, T\right] \times \mathcal{O}$, the random variable $u(t, x)$ is absolutely continuous with respect to the Lebesgue measure.

(b) Moreover, if for some integer $m \geq 2, f \in C_{\mathrm{pol}}^{m}(\mathbb{R})$, then the density of $u(t, x)$ belongs to $C^{m-2}(\mathbb{R})$. 
As we shall see below, the assumption (5.1) is not needed to prove Malliavin regularity of $u(t, x)$. It is instead needed to prove finiteness of negative moments of the Malliavin matrix (which reduces to a real random variable in the present setting). Moreover, as explained in Remark 5.9, in order to prove the existence of density, condition (5.1) can be slightly weakened. Nevertheless, for the sake of conciseness and clarity, we have decided to state only one condition of the term $g(x, t)$.

Remark 5.2. The hypothesis on the initial datum in the previous theorem may be relaxed to $u_{0} \in \mathbb{L}^{q}(C(\overline{\mathcal{O}}))$ for all $q \geq 1$, and $u_{0} \in L^{\infty}\left(\mathcal{O} \rightarrow \mathbb{D}^{1, \infty}\right)$. However, it does not seem natural to assume the initial datum to have such regularity.

Let us give some examples of domains $\mathcal{O}$ and covariance operators $Q=B B^{*}$ satisfying condition (5.1) above.

Example 5.3. Let $d=1, \mathcal{O}=(0,1)$ and $B=$ Id. Then (5.1) holds with $\gamma=\frac{1}{2}$ (see e.g. [1, (A.3)]).

Example 5.4. Let $\mathcal{O}=(0, \pi)^{d}$. Define, for any $k=\left(k_{1}, \ldots, k_{d}\right) \in \mathbb{N}^{d}$,

$$
e_{k}(x):=\left(\frac{2}{\pi}\right)^{\frac{d}{2}} \sin \left(k_{1} x_{1}\right) \cdots \sin \left(k_{d} x_{d}\right), \quad x \in \mathcal{O} .
$$

Then, it is readily checked that the family $\left\{e_{k}\right\}_{k \in \mathbb{N}^{d}}$ is an orthonormal basis of $L^{2}(\mathcal{O})$ such that

$$
-\Delta e_{k}=|k|^{2} e_{k}
$$

where $|k|^{2}:=k_{1}^{2}+\cdots+k_{d}^{2}$. Set $B=(I-\Delta)^{-m}$ for $m \geq 0$. Then, since $Q=(I-\Delta)^{-2 m}$, one has

$$
\begin{aligned}
g(x, t) & =\int_{0}^{t} \int_{\mathcal{O}} G_{s}(x, y)\left[Q G_{s}(x, \cdot)\right](y) d y d s \\
& =\int_{0}^{t} \sum_{k \in \mathbb{N}^{d}}\left(1+|k|^{2}\right)^{-m}\left\langle G_{s}(x, \cdot), e_{k}\right\rangle_{L^{2}}^{2} d s \\
& =\int_{0}^{t} \sum_{k \in \mathbb{N}^{d}}\left(1+|k|^{2}\right)^{-m} e^{-2 s|k|^{2}}\left|e_{k}(x)\right|^{2} d s \\
& =\frac{1}{2} \sum_{k \in \mathbb{N}^{d}}\left(1+|k|^{2}\right)^{-m}|k|^{-2}\left(1-e^{-2 t|k|^{2}}\right)\left|e_{k}(x)\right|^{2} .
\end{aligned}
$$

Using the fact that $\left|e_{k}(x)\right|$ is uniformly bounded with respect to $k$ and $x$, one easily verifies that $m>\frac{d}{2}-1$ implies that the latter series is finite. Moreover, we have that

$$
1-e^{-2 t|k|^{2}} \geq \frac{2 t|k|^{2}}{1+2 t|k|^{2}} \geq \frac{2 t|k|^{2}}{1+2 T|k|^{2}} .
$$

Hence

$$
g(x, t) \geq t \sum_{k \in \mathbb{N}^{d}}\left(1+|k|^{2}\right)^{-m}\left(1+2 T|k|^{2}\right)^{-1}\left|e_{k}(x)\right|^{2}
$$


and this series can be bounded from below by any of its summands, such as the corresponding to $k=(1, \ldots, 1) \in \mathbb{N}^{d}$. Therefore,

$$
g(x, t) \geq c_{x} t, \quad \text { with } \quad c_{x}:=(1+d)^{-m}(1+2 T d)^{-1}\left(\frac{2}{\pi}\right)^{\frac{d}{2}} \sin \left(x_{1}\right) \cdots \sin \left(x_{d}\right) .
$$

Since $x \in(0, \pi)^{d}$, it is clear that $c_{x}>0$ and this implies that condition (5.1) is fulfilled with $\gamma=1$.

Before turning to the study of the Malliavin differentiability of the solution to our equation, let us recall that the underlying Gaussian space on which to perform Malliavin calculus is given by the isonormal Gaussian process on the Hilbert space $H:=$ $L^{2}\left([0, T] \rightarrow L_{Q}^{2}\right)$, which can be naturally associated to the cylindrical Wiener process $\bar{W}$ with covariance $Q$. With a slight (but harmless) abuse of notation we shall write $W$ instead of $\bar{W}$ for notational convenience.

5.1. Malliavin differentiability. The purpose of this subsection is to prove regularity of the collection of random variables $\{u(t, x)\}_{(t, x) \in \mathcal{O}_{T}}$ in the sense of Malliavin. Proposition 5.5 concerns the Malliavin differentiability of order one, while Proposition 5.7 treats higher-order Malliavin derivatives. As already mentioned, the two results rely on hypotheses (a) and (b) of Theorem 5.1, respectively, but not on the lower bound for the stochastic convolution.

Proposition 5.5. Assume that

(i) $u_{0} \in C(\overline{\mathcal{O}})$;

(ii) $f$ is increasing and belongs to $C_{\text {pol }}^{1}(\mathbb{R})$;

(iii) $Q$ is positivity preserving.

Let $u \in \bigcap_{q \geq 1} \mathbb{C}_{q}$ be the mild solution to (2.1). Then, for any $(t, x) \in \mathcal{O}_{T}$, one has $u(t, x) \in \mathbb{D}^{1, \infty}$. Moreover, the Malliavin derivative $D u(t, x)$ satisfies the following linear equation in $H$ :

$$
D u(t, x)=v_{0}(t, x)+\int_{0}^{t} \int_{\mathcal{O}} G_{t-s}(x, y)\left(\eta-f^{\prime}(u(s, y))\right) D u(s, y) d y d s
$$

where $v_{0}(t, x):=(\tau, z) \mapsto G_{t-\tau}(x, z) 1_{[0, t]}(\tau)$, and it holds that, for all $q \geq 1$,

$$
\sup _{(t, x) \in \mathcal{O}_{T}} \mathbb{E}\|D u(t, x)\|_{H}^{q}<\infty .
$$

Proof. Since $f_{\lambda}$ is Lipschitz continuous and of class $C^{1}$, slight modifications of the "classical" results (cf. e.g. [18]) imply that, for any $(t, x) \in \mathcal{O}_{T}, u_{\lambda}(t, x)$ belongs to $\mathbb{D}^{1, \infty}$ and satisfies the following linear deterministic integral equation with random coefficients:

$$
D u_{\lambda}(t, x)=v_{0}(t, x)+\int_{0}^{t} \int_{\mathcal{O}} G_{t-s}(x, y)\left(\eta-f_{\lambda}^{\prime}\left(u_{\lambda}(s, y)\right)\right) D u_{\lambda}(s, y) d y d s .
$$

Recall that, by Proposition 2.5, one has, for any $q \geq 1$,

$$
\mathbb{E}\left|u_{\lambda}(t, x)-u(t, x)\right|^{q} \rightarrow 0
$$


as $\lambda \rightarrow 0$, for all $(t, x) \in \mathcal{O}_{T}$. Therefore, by Lemma 4.1, in order to conclude that $u(t, x)$ belongs to $\mathbb{D}^{1, \infty}$ for all $(t, x) \in \mathcal{O}_{T}$, it suffices to show that for all $q \geq 1$ and $(t, x) \in \mathcal{O}_{T}$, one has

$$
\sup _{\lambda>0} \mathbb{E}\left\|D u_{\lambda}(t, x)\right\|_{H}^{q}<\infty .
$$

Let $\left\{h^{k}\right\}_{k \in \mathbb{N}}$ be an orthonormal basis of $H$, and set

$$
\varphi_{\lambda}^{k}(t, x):=\left\langle D u_{\lambda}(t, x), h^{k}\right\rangle_{H}, \quad(t, x) \in \mathcal{O}_{T} .
$$

Then $\varphi_{\lambda}^{k}(t):=\varphi_{\lambda}^{k}(t, \cdot), 0 \leq t \leq T$, satisfies the deterministic evolution equation with random coefficients

$$
\frac{d}{d t} \varphi_{\lambda}^{k}(t)-\Delta \varphi_{\lambda}^{k}(t)+F_{\lambda}(t) \varphi_{\lambda}^{k}(t)=\Phi^{k}(t), \quad \varphi_{\lambda}^{k}(0)=0,
$$

where $F_{\lambda}(t):=f_{\lambda}^{\prime}\left(u_{\lambda}(t, \cdot)\right)-\eta$ and $\Phi^{k}(t):=Q h^{k}(t)$. In fact, one has

$$
\begin{aligned}
\left\langle v_{0}(t, x), h^{k}\right\rangle_{H} & =\int_{0}^{t}\left\langle G_{t-s}(x, \cdot), h^{k}(s)\right\rangle_{L_{Q}^{2}} d s=\int_{0}^{t} \int_{\mathcal{O}} G_{t-s}(x, y)\left[Q h^{k}(s)\right](y) d y d s \\
& =\left[\int_{0}^{t} S(t-s) Q h^{k}(s) d s\right](x) .
\end{aligned}
$$

From now we assume, without loss of generality, that $\eta=0$ (if not, it is enough to write the corresponding equation for $t \mapsto e^{-\eta t} \varphi_{\lambda}(t)$, multiplying by $e^{-\eta t}$ the term $\Phi^{k}(t)$ ).

Fix $\omega \in \Omega$, and let $(s, t) \mapsto U_{\lambda}(t, s), s \leq t$, denote the family of evolution operators generated by the time-dependent linear operator $\Delta-F_{\lambda}(t)$. Then we can write

$$
\varphi_{\lambda}^{k}(t)=\int_{0}^{t} U_{\lambda}(t, s) \Phi^{k}(s) d s
$$

thus also, denoting the integral kernel of $U_{\lambda}(t, s)$ (that exists by Proposition 4.3 (iv)), by $k_{\lambda}(t, s ; \cdot, \cdot)$,

$$
\varphi_{\lambda}^{k}(t, x)=\int_{0}^{t} \int_{\mathcal{O}} k_{\lambda}(t, s ; x, y) \Phi^{k}(s, y) d y d s
$$

This yields

$$
\begin{aligned}
\left\|D u_{\lambda}(t, x)\right\|_{H}^{2} & =\sum_{k \in \mathbb{N}}\left|\varphi_{\lambda}^{k}(t, x)\right|^{2}=\sum_{k \in \mathbb{N}}\left|\int_{0}^{t} \int_{\mathcal{O}} k_{\lambda}(t, s ; x, y) \Phi^{k}(s, y) d y d s\right|^{2} \\
& =\sum_{k \in \mathbb{N}}\left|\left\langle k_{\lambda}(t, \cdot ; x, \cdot) 1_{[0, t]}(\cdot), h^{k}\right\rangle_{L^{2}\left([0, T] \rightarrow L_{Q}^{2}\right)}\right|^{2} \\
& =\int_{0}^{t}\left\|k_{\lambda}(t, s ; x, \cdot)\right\|_{L_{Q}^{2}}^{2} d s .
\end{aligned}
$$

Note that Proposition 4.3(ii) implies $k_{\lambda}(t, s) \leq G_{t-s}$ pointwise for all $0 \leq s<t \leq T$. Using that $Q$ is positivity preserving, we are left with

$$
\left\|D u_{\lambda}(t, x)\right\|_{H}^{2} \leq \int_{0}^{t}\left\|G_{t-s}(x, \cdot)\right\|_{L_{Q}^{2}}^{2} d s=\left\|v_{0}(t, x)\right\|_{H}^{2} .
$$


Let us now show that $\left\|v_{0}(t, x)\right\|_{H}^{2}$ is uniformly bounded over $t$ and $x$. In fact, one has

$$
\begin{aligned}
\left\|v_{0}(t, x)\right\|_{H}^{2} & =\int_{0}^{t}\left\|G_{t-\tau}(x, \cdot)\right\|_{L_{Q}^{2}}^{2} d \tau=\int_{0}^{t}\left\|B^{*} G_{t-\tau}(x, \cdot)\right\|_{2}^{2} d \tau \\
& =\int_{0}^{t} \sum_{k \in \mathbb{N}}\left\langle G_{t-\tau}(x, \cdot), B e^{k}\right\rangle_{2}^{2} d \tau=\int_{0}^{t} \sum_{k \geq 1}\left(\int_{\mathcal{O}} G_{t-\tau}(x, y)\left[B e^{k}\right](y) d y\right)^{2} d \tau \\
& =\sum_{k \in \mathbb{N}} \int_{0}^{t}\left[S(t-\tau) B e^{k}\right](x)^{2} d \tau,
\end{aligned}
$$

where $\left\{e^{k}\right\}_{k \in \mathbb{N}}$ is an orthonormal basis of $L^{2}$. The identities

$$
\mathbb{E}\left|W_{A}(t, x)\right|^{2}=\mathbb{E}\left|\sum_{k \in \mathbb{N}} \int_{0}^{t}\left[S(t-s) B e^{k}\right](x) d w_{k}(s)\right|^{2}=\sum_{k \in \mathbb{N}} \int_{0}^{t}\left[S(t-s) B e^{k}\right](x)^{2} d s,
$$

yield $\left\|v_{0}(t, x)\right\|_{H}^{2}=\mathbb{E}\left|W_{A}(t, x)\right|^{2}$ for all $(t, x) \in \mathcal{O}_{T}$. Thanks to Hypothesis 2.3 we infer that there exists a constant $N=N(q)$, independent of $\lambda$, such that

$$
\sup _{(t, x) \in \mathcal{O}_{T}} \mathbb{E}\left\|D u_{\lambda}(t, x)\right\|_{H}^{q}<N
$$

We have thus proved that $u(t, x) \in \mathbb{D}^{1, \infty}$ for all $(t, x) \in \mathcal{O}_{T}$. It is therefore lawful to apply the Malliavin derivative to the equation satisfied by $u$, obtaining

$$
D u(t, x)=v_{0}(t, x)-\int_{0}^{t} \int_{\mathcal{O}} G_{t-s}(x, y) D f(u(s, y) d y d s .
$$

Then, appealing to the chain rule proved in Lemma 4.2, we obtain that the Malliavin derivative $D u(t, x)$ satisfies equation (5.2).

In order to conclude, we only have to show that estimate (5.3) holds true. The argument is essentially the same as above, hence it is only sketched. Still assuming $\eta=0$ without loss of generality, setting $F(t, x):=f^{\prime}(u(t, x))$, one has that $\varphi^{k}(t, x):=$ $\left\langle D u(t, x), h^{k}\right\rangle_{H}$ satisfies the linear deterministic evolution equation with random coefficients

$$
\frac{d}{d t} \varphi^{k}(t)-\Delta \varphi^{k}(t)+F(t) \varphi^{k}(t)=\Phi^{k}(t), \quad \varphi^{k}(0)=0 .
$$

Let $\Omega^{\prime} \subset \Omega$ with $\mathbb{P}\left(\Omega^{\prime}\right)=1$ such that $(t, x) \mapsto u(t, x, \omega) \in C([0, T] \times \overline{\mathcal{O}})$ for all $\omega \in \Omega^{\prime}$. Fix $\omega \in \Omega^{\prime}$. Then $(t, x) \mapsto F(t, x)$ is positive and continuous, hence bounded on the compact set $[0, T] \times \overline{\mathcal{O}}$. One can then construct the evolution operator associated to $\Delta-F$, and proceeding exactly as above one arrives at

$$
\sup _{(t, x) \in \mathcal{O}_{T}} \mathbb{E}\|D u(t, x)\|_{H}^{q}<\infty
$$

so that the proof is complete.

Remark 5.6. Condition (iii) in Proposition 5.5 above does not need to be considered an important restriction. Indeed, this condition is satisfied in the spatially homogeneous counterpart when dealing with existence and smoothness of the density for stochastic heat and wave equations in $\mathbb{R}^{d}$ (see e.g. [19]).

Proposition 5.7. Let $m \in \mathbb{N}, m \geq 2$. Assume that 
(i) $u_{0} \in C(\overline{\mathcal{O}})$;

(ii) $f$ is increasing and belongs to $C_{\mathrm{pol}}^{m}(\mathbb{R})$;

(iii) $Q$ is positivity preserving.

Let $u \in \cap_{q \in \mathbb{N}} \mathbb{C}_{q}$ be the unique mild solution to (2.1). Then, for any $(t, x) \in \mathcal{O}_{T}$, one has $u(t, x) \in \mathbb{D}^{m, \infty}$ and

$$
\sup _{(t, x) \in \mathcal{O}_{T}}\|u(t, x)\|_{\mathbb{D}^{m, q}}<\infty \quad \forall q \geq 1 .
$$

Proof. Let $p \in \mathbb{N}$ be such that $\left.|f(x)+| f^{\prime}(x)|+\cdots+| f^{(m)}(x)|\lesssim 1+| x\right|^{p}$ for all $r \in \mathbb{R}$. By Proposition 5.5 , we have that $u(t, x) \in \mathbb{D}^{1, \infty}$ for all $(t, x) \in \mathcal{O}_{T}$. Let us consider the regularized equation (2.4): since $f_{\lambda}$ needs not have bounded derivatives of order two and higher, we cannot apply "classical" results (cf. e.g. [18]) to deduce that, for any $(t, x) \in \mathcal{O}_{T}, u_{\lambda}(t, x)$ belongs to $\mathbb{D}^{m, \infty}$. For this reason, we introduce a further regularization: let $\left\{\zeta_{\beta}\right\}_{\beta}$ be a family of mollifiers as in Lemma 4.5 above. Note that $f_{\lambda \beta}^{(n)}=f_{\lambda}^{\prime} * \zeta_{\beta}^{(n-1)}$ for all $n \geq 1$, hence $f_{\lambda \beta}$ has bounded derivatives of every order. Let $u_{\lambda \beta}$ be the unique mild solution in $\cap_{q \in \mathbb{N}} \mathbb{C}_{q}$ to the equation

$$
d u_{\lambda \beta}(t)-\Delta u_{\lambda \beta}(t) d t+f_{\lambda \beta}\left(u_{\lambda \beta}(t)\right) d t=\eta u_{\lambda \beta}(t) d t+B d W(t), \quad u_{\lambda \beta}(0)=u_{0} .
$$

We split the rest of the proof in three steps: first we show that, for any $(t, x) \in \mathcal{O}_{T}$, one has $u_{\lambda \beta}(t, x) \rightarrow u(t, x)$ in $\mathbb{L}^{q}$ as $\beta \rightarrow 0$. Then we obtain the uniform bound

$$
\sup _{(t, x) \in \mathcal{O}_{T}} \mathbb{E}\left\|D^{n} u_{\lambda \beta}(t, x)\right\|_{H^{\otimes n}}<N,
$$

where $N$ is a constant independent of $\lambda$ and $\beta$. Finally we pass to the limit as $\beta \rightarrow 0$ and $\lambda \rightarrow 0$.

Step 1. We assume again, without loss of generality, that $\eta=0$. It is easily seen that it holds

$$
u_{\lambda \beta}(t)-u_{\lambda}(t)=\int_{0}^{t} S(t-s)\left(f_{\lambda}\left(u_{\lambda}(s)\right)-f_{\lambda \beta}\left(u_{\lambda \beta}(s)\right)\right) d s,
$$

hence, recalling that $S(t)$ is contracting in $L^{\infty}(\mathcal{O})$ and denoting the norm of this space by $\|\cdot\|$,

$$
\left\|u_{\lambda \beta}(t)-u_{\lambda}(t)\right\| \leq \int_{0}^{t}\left\|f_{\lambda}\left(u_{\lambda}(s)\right)-f_{\lambda \beta}\left(u_{\lambda \beta}(s)\right)\right\| d s .
$$

This yields, by the triangle inequality,

$$
\begin{aligned}
\left\|u_{\lambda \beta}(t)-u_{\lambda}(t)\right\| & \leq \int_{0}^{t}\left\|f_{\lambda \beta}\left(u_{\lambda \beta}(s)\right)-f_{\lambda \beta}\left(u_{\lambda}(s)\right)\right\| d s+\int_{0}^{t}\left\|f_{\lambda \beta}\left(u_{\lambda}(s)\right)-f_{\lambda}\left(u_{\lambda}(s)\right)\right\| d s \\
& \leq \frac{1}{\lambda} \int_{0}^{t}\left\|u_{\lambda \beta}(s)-u_{\lambda}(s)\right\| d s+I_{\beta},
\end{aligned}
$$

where

$$
I_{\beta}:=\int_{0}^{T}\left\|f_{\lambda \beta}\left(u_{\lambda}(s)\right)-f_{\lambda}\left(u_{\lambda}(s)\right)\right\| d s .
$$

By Gronwall's inequality and some obvious manipulations, one arrives at

$$
\mathbb{E} \sup _{t \leq T}\left\|u_{\lambda \beta}(t)-u_{\lambda}(t)\right\|^{q} \leq e^{q T / \lambda} \mathbb{E} I_{\beta}^{q} .
$$


Let us show that $\mathbb{E} I_{\beta}^{q} \rightarrow 0$ as $\beta \rightarrow 0$ : since $f_{\lambda}$ is continuous, $f_{\lambda \beta}$ converges uniformly to $f_{\lambda}$ as $\beta \rightarrow 0$. Therefore, as $u_{\lambda}(s) \in C(\overline{\mathcal{O}}) \mathbb{P}$-a.s., we also have that the integrand in the definition of $I_{\beta}$ converges to zero $\mathbb{P}$-a.s. as $\beta \rightarrow 0$. Taking into account that

$$
\left\|f_{\lambda \beta}\left(u_{\lambda}(s)\right)-f_{\lambda}\left(u_{\lambda}(s)\right)\right\|^{q} \lesssim_{\lambda} 1+\left\|u_{\lambda}(s)\right\|^{q},
$$

and that $\mathbb{E} \int_{0}^{T}\left\|u_{\lambda}(s)\right\|^{q} d s<\infty$, we get, by the dominated convergence theorem, that $\mathbb{E} I_{\beta}^{q} \rightarrow 0$, hence also

$$
\mathbb{E} \sup _{t \leq T}\left\|u_{\lambda \beta}(t)-u_{\lambda}(t)\right\|_{C(\overline{\mathcal{O}})}^{q} \rightarrow 0
$$

as $\beta \rightarrow 0$, for any $q \geq 1$.

Step 2. For $n=1$ it is easily seen that (5.8) holds true, simply by the previous proposition, noting that $f_{\lambda \beta}^{\prime}=f_{\lambda}^{\prime} * \zeta_{\beta} \geq 0$. For the sake of clarity, let us explicitly show, in the case $n=2$, how estimate (5.8) is implied by the corresponding one with $n=1$. Then the general induction step will be clear. As before, we shall assume, without loss of generality, that $\eta=0$. Since, as already observed before, $f_{\lambda \beta}$ has bounded derivatives of every order, we infer that $u_{\lambda \beta}(t, x) \in \mathbb{D}^{2, \infty}$, the iterated Malliavin derivative $D^{2} u_{\lambda \beta}(t, x)$ takes values in $H^{\otimes 2}$ and satisfies

$$
\begin{aligned}
D^{2} u_{\lambda \beta}(t, x) & +\int_{0}^{t} \int_{\mathcal{O}} G_{t-s}(x, y) f_{\lambda \beta}^{\prime \prime}\left(u_{\lambda \beta}(s, y)\right)\left(D u_{\lambda \beta}(s, y)\right)^{\otimes 2} d y d s \\
& \left.+\int_{0}^{t} \int_{\mathcal{O}} G_{t-s}(x, y) f_{\lambda \beta}^{\prime}\left(u_{\lambda \beta}(s, y)\right)\right) D^{2} u_{\lambda \beta}(s, y) d y d s=0 .
\end{aligned}
$$

Let $\left\{h^{k}\right\}_{k \in \mathbb{N}}$ be an orthonormal basis of $H^{\otimes 2}$ and set

$$
\varphi_{\lambda \beta}^{k}(t, x):=\left\langle D^{2} u_{\lambda \beta}(t, x), h^{k}\right\rangle_{H^{\otimes 2}}, \quad k \in \mathbb{N}
$$

Then $\varphi_{\lambda \beta}^{k}(t):=\varphi_{\lambda \beta}^{k}(t, \cdot)$ satisfies the following linear deterministic evolution equation with random coefficients

$$
\frac{d}{d t} \varphi_{\lambda \beta}^{k}(t)-\Delta \varphi_{\lambda \beta}^{k}(t)+F_{\lambda \beta}(t) \varphi_{\lambda \beta}^{k}(t)=\Phi_{\lambda \beta}^{k}(t), \quad \varphi_{\lambda \beta}^{k}(0)=0
$$

where

$$
F_{\lambda \beta}(t):=f_{\lambda \beta}^{\prime}\left(u_{\lambda \beta}(t, \cdot)\right), \quad \Phi_{\lambda \beta}^{k}(t):=f_{\lambda \beta}^{\prime \prime}\left(u_{\lambda \beta}(t, \cdot)\right)\left\langle\left(D u_{\lambda \beta}(t, \cdot)\right)^{\otimes 2}, h^{k}\right\rangle_{H^{\otimes 2}} .
$$

Then we have

$$
\varphi_{\lambda \beta}^{k}(t)=\int_{0}^{t} U_{\lambda \beta}(t, s) \Phi_{\lambda \beta}^{k}(s) d s
$$


hence also, denoting the kernel of $U_{\lambda \beta}(t, s)$ by $k(t, s ; \cdot, \cdot)$,

$$
\begin{aligned}
\left|\varphi_{\lambda \beta}^{k}(t, x)\right|^{2} & =\left|\int_{0}^{t} \int_{\mathcal{O}} k_{\lambda \beta}(t, s ; x, y) \Phi_{\lambda \beta}^{k}(s, y) d y d s\right|^{2} \\
& \leq\left(\int_{0}^{t} \int_{\mathcal{O}} k_{\lambda \beta}(t, s ; x, y)\left|\Phi_{\lambda \beta}^{k}(s, y)\right| d y d s\right)^{2} \\
& \leq\left(\int_{0}^{t} \int_{\mathcal{O}} G_{t-s}(x, y)\left|\Phi_{\lambda \beta}^{k}(s, y)\right| d y d s\right)^{2} \\
& \lesssim_{T} \int_{0}^{t} \int_{\mathcal{O}} G_{t-s}(x, y)\left|\Phi_{\lambda \beta}^{k}(s, y)\right|^{2} d y d s,
\end{aligned}
$$

where we have used the estimate $k_{\lambda \beta} \leq G$ in the first inequality, and Cauchy-Schwarz' inequality in the third inequality, recalling that $S(t)$ is contracting in $L^{\infty}$ (cf. (3.1)).

Summing over $k$, Tonelli's theorem yields

$$
\begin{aligned}
\left\|D^{2} u_{\lambda \beta}(t, x)\right\|_{H^{\otimes 2}}^{2} & =\sum_{k \in \mathbb{N}}\left|\varphi_{\lambda \beta}^{k}(t, x)\right|^{2} \lesssim_{T} \int_{0}^{t} \int_{\mathcal{O}} G_{t-s}(x, y) \sum_{k \in \mathbb{N}}\left|\Phi_{\lambda \beta}^{k}(s, y)\right|^{2} d y d s \\
& =\int_{0}^{t} \int_{\mathcal{O}} G_{t-s}(x, y)\left\|\Phi_{\lambda \beta}(s, y)\right\|_{H \otimes 2}^{2} d y d s .
\end{aligned}
$$

where

$$
\Phi_{\lambda \beta}(t, x):=f_{\lambda \beta}^{\prime \prime}\left(u_{\lambda \beta}(t, x)\right)\left(D u_{\lambda \beta}(t, x)\right)^{\otimes 2} .
$$

Hölder's inequality and Tonelli's theorem then imply

$$
\mathbb{E}\left\|D^{2} u_{\lambda \beta}(t, x)\right\|_{H^{\otimes 2}}^{q} \lesssim_{T} \int_{0}^{t} \int_{\mathcal{O}} G_{t-s}(x, y) \mathbb{E}\left\|\Phi_{\lambda \beta}(s, y)\right\|_{H^{\otimes 2}}^{q} d y d s,
$$

that is,

$$
\sup _{(t, x) \in \mathcal{O}_{T}} \mathbb{E}\left\|D^{2} u_{\lambda \beta}(t, x)\right\|_{H \otimes 2}^{q} \lesssim_{T} \sup _{(t, x) \in \mathcal{O}_{T}} \mathbb{E}\left\|\Phi_{\lambda \beta}(t, x)\right\|_{H \otimes 2}^{q}
$$

Let us show that the right-hand side is finite: by Cauchy-Schwarz' inequality, we have

$$
\mathbb{E}\left\|\Phi_{\lambda \beta}(t, x)\right\|_{H^{\otimes 2}}^{q} \lesssim\left(\mathbb{E}\left|f_{\lambda \beta}^{\prime \prime}\left(u_{\lambda \beta}(t, x)\right)\right|^{2 q}\right)^{1 / 2}\left(\mathbb{E}\left\|D u_{\lambda \beta}(t, x)\right\|_{H}^{4 q}\right)^{1 / 2}
$$

Assume, without loss of generality, $\lambda \leq 1, \beta \leq 1$. Since $f_{\lambda \beta}^{\prime \prime}=f_{\lambda}^{\prime \prime} * \zeta_{\beta}$, and, by Lemma 4.4. there exists $\sigma \in \mathbb{N}$ such that $\left|f_{\lambda}^{\prime \prime}(x)\right| \lesssim 1+|x|^{\sigma}$, by Lemma 4.5 we also have $\left|f_{\lambda \beta}^{\prime \prime}(x)\right| \lesssim 1+|x|^{\sigma}$. Therefore

$$
\mathbb{E}\left\|\Phi_{\lambda \beta}(t, x)\right\|_{H^{\otimes 2}}^{q} \lesssim\left(1+\mathbb{E}\left|u_{\lambda \beta}(t, x)\right|^{2 q \sigma}\right)^{1 / 2}\left(\mathbb{E}\left\|D u_{\lambda \beta}(t, x)\right\|_{H}^{4 q}\right)^{1 / 2}
$$

where both terms on the right hand side are uniformly bounded over $t, x, \lambda$, and $\beta$ by results already proved; in fact, as for $u_{\lambda}$ itself, the boundedness of the first term on the right-hand side above follows from Proposition 6.2.2 in [4], since $f_{\lambda \beta}$ is also monotone. The claim is then verified for $n=2$.

The general case is proved by induction in a completely similar way. In particular, assume that, given $3 \leq n<m$, one has the uniform bound

$$
\sup _{(t, x) \in \mathcal{O}_{T}}\left\|u_{\lambda \beta}(t, x)\right\|_{\mathbb{D}^{n-1, q}}<N,
$$


with $N$ independent of $\lambda$ and $\beta$. Let $\left\{h^{k}\right\}_{k \in \mathbb{N}}$ be an orthonormal basis of $H^{\otimes n}$, and set

$$
\varphi_{\lambda \beta}^{k}(t, x):=\left\langle D^{n} u_{\lambda \beta}(t, x), h^{k}\right\rangle_{H \otimes n} .
$$

Then $\varphi^{k}(t, \cdot)$ satisfies an equation of the form (5.9), where $\Phi^{k}$ is a sum of finitely many terms depending on $u_{\lambda \beta}$ and on its Malliavin derivatives of order not greater than $n-1$, whence

$$
\sup _{(t, x) \in \mathcal{O}_{T}} \mathbb{E}\left\|\Phi_{\lambda \beta}(t, x)\right\|_{H^{\otimes n}}^{q}<N
$$

with $N$ a constant that does not depend on $\lambda$ nor on $\beta$. Moreover, by an argument completely analogous to one already used before, one shows that

$$
\left.\sup _{(t, x) \in \mathcal{O}_{T}} \mathbb{E}\left\|D^{n} u_{\lambda \beta}(t, x)\right\|_{H \otimes n}^{q} \lesssim \sup _{(t, x) \in \mathcal{O}_{T}} \mathbb{E} \| \Phi_{\lambda \beta}(t, x)\right) \|_{H \otimes n}^{q}<N
$$

where $N$ is the same constant of the previous inequality.

Step 3. Let $q>1$. By the previous steps and Lemma 4.1. passing to the limit as $\beta \rightarrow 0$, we obtain $u_{\lambda}(t, x) \in \mathbb{D}^{m, q}$ for all $(t, x) \in \mathcal{O}_{T}$, and also, by lower semicontinuity of the norm with respect to weak convergence,

$$
\mathbb{E}\left\|D^{n} u_{\lambda}(t, x)\right\|_{H_{\otimes n}}^{q} \leq \liminf _{\beta \rightarrow 0} \mathbb{E}\left\|D^{n} u_{\lambda \beta}(t, x)\right\|_{H^{\otimes n}}^{q},
$$

which implies, together with the last inequality,

$$
\sup _{(t, x) \in \mathcal{O}_{T}} \mathbb{E}\left\|D^{n} u_{\lambda}(t, x)\right\|_{H \otimes n}^{q}<N .
$$

Recalling that, by Proposition 2.5. $u_{\lambda}(t, x) \rightarrow u(t, x)$ in $\mathbb{L}^{q}$ as $\lambda \rightarrow 0$ for all $(t, x) \in \mathcal{O}_{T}$, appealing again to Lemma 4.1. we arrive at $u(t, x) \in \mathbb{D}^{m, q}$ for all $(t, x)$. Since $q$ is arbitrary, we conclude that $u(t, x) \in \mathbb{D}^{m, \infty}$ for all $(t, x)$.

5.2. Analysis of the Malliavin matrix. In this subsection, we shall use a standard method in order to prove that the inverse of the Malliavin matrix has moments of all orders (see e.g. [19, Theorem 6.2]).

Proposition 5.8. Assume that the hypotheses of Theorem 5.1(a) are satisfied, as well as condition (5.1). Then, for any $(t, x) \in] 0, T] \times \mathcal{O}$, one has

$$
\mathbb{E}\|D u(t, x)\|_{H}^{-q}<\infty \quad \forall q \geq 1 .
$$

Proof. By [17, Lemma 2.3.1], it suffices to prove that for any $q \geq 2$, there exists $\varepsilon_{0}(q)>0$ such that, for all $\varepsilon \leq \varepsilon_{0}$,

$$
\mathbb{P}\left(\|D u(t, x)\|_{H}^{2}<\varepsilon\right) \lesssim \varepsilon^{q} .
$$

Let $(t, x) \in] 0, T] \times \mathcal{O}$ be fixed. Observe that we are assuming the same hypotheses as in Proposition [5.5. Hence, $u(t, x)$ belongs to $\mathbb{D}^{1, \infty}$ and the Malliavin derivative $D u(t, x)$ satisfies equation (5.2). Then, using this latter equation, we can infer that, for any $\delta>0$ sufficiently small,

$$
\|D u(t, x)\|_{H}^{2}=\int_{0}^{t}\left\|D_{\tau} u(t, x)\right\|_{L_{Q}^{2}}^{2} d \tau \geq \int_{t-\delta}^{t}\left\|D_{\tau} u(t, x)\right\|_{L_{Q}^{2}}^{2} d \tau \geq \frac{1}{2} g(x, \delta)-I(t, x, \delta),
$$


where $g(x, \delta)$ is as in assumption (5.1) and

$$
I(t, x, \delta):=\int_{0}^{\delta}\left\|\int_{t-\tau}^{t} \int_{\mathcal{O}} G_{t-s}(x, y)\left(\eta-f^{\prime}(u(s, y))\right) D_{t-\tau} u(s, y) d y d s\right\|_{L_{Q}^{2}}^{2} d \tau .
$$

Hence, using Chebyshev's inequality, we have, for all $\varepsilon>0$,

$$
\mathbb{P}\left(\|D u(t, x)\|_{H}^{2}<\varepsilon\right) \leq \mathbb{P}\left\{I(t, x ; \delta) \geq \frac{g(x, \delta)}{2}-\varepsilon\right\} \leq\left(\frac{g(x, \delta)}{2}-\varepsilon\right)^{-p} \mathbb{E}|I(t, x, \delta)|^{p} .
$$

Let us now find an upper bound for the $p$-th moment of $I(t, x, \delta)$. For this, we start by applying Minkowski and Hölder's inequalities, the latter with respect to the measure on $[t-\delta, t] \times \mathcal{O}$ given by $G_{t-s}(x, y) d y d s$, to obtain that

$$
\begin{aligned}
\mathbb{E}|I(t, x, \delta)|^{p} \leq \mathbb{E}\left(\int_{t-\delta}^{t} \int_{\mathcal{O}} G_{t-s}(x, y)\left|\eta-f^{\prime}(u(s, y))\right|\left\|D_{t-.} u(s, y)\right\|_{L^{2}\left([0, \delta] ; L_{Q}^{2}\right)} d y d s\right)^{2 p} \\
\leq\left(\int_{t-\delta}^{t} \int_{\mathcal{O}} G_{t-s}(x, y) d y d s\right)^{2 p-1} \\
\quad \times \int_{t-\delta}^{t} \int_{\mathcal{O}} G_{t-s}(x, y) \mathbb{E}\left(\left(\eta+\left|f^{\prime}(u(s, y))\right|\right)^{2 p}\left\|D_{t-.} u(s, y)\right\|_{L^{2}\left([0, \delta] ; L_{Q}^{2}\right)}^{2 p}\right) d y d s \\
\lesssim \delta^{2 p-1} \int_{t-\delta}^{t} \int_{\mathcal{O}} G_{t-s}(x, y) \mathbb{E}\left(\left(\eta+\left|f^{\prime}(u(s, y))\right|\right)^{2 p}\left\|D_{t-.} u(s, y)\right\|_{L^{2}\left([0, \delta] ; L_{Q}^{2}\right)}^{2 p}\right) d y d s
\end{aligned}
$$

where we have also used the estimate (3.1). Thus, applying the Cauchy-Schwarz inequality and appealing to the polynomial growth condition on $f^{\prime}$ (say $\left|f^{\prime}(z)\right| \lesssim 1+|z|^{r}$ for all $z \in \mathbb{R}$ ), the right hand side of (5.12) can be estimated, up to a positive constant, by

$$
\begin{aligned}
& \delta^{2 p-1} \sup _{(s, y) \in[t-\delta, t] \times \mathcal{O}}\left(\mathbb{E}\left\|D_{t-.} u(s, y)\right\|_{L^{2}\left([0, \delta] ; L_{Q}^{2}\right)}^{4 p}\right)^{1 / 2} \\
& \times \int_{t-\delta}^{t} \int_{\mathcal{O}} G_{t-s}(x, y)\left(1+\left(\mathbb{E}|u(s, y)|^{4 r p}\right)^{1 / 2}\right) d y d s .
\end{aligned}
$$

At this point, we can appeal to (5.3) to get

$$
\sup _{(s, y) \in[t-\delta, t] \times \mathcal{O}}\left(\mathbb{E}\left\|D_{t-.} . u(s, y)\right\|_{L^{2}\left([0, \delta] ; L_{Q}^{2}\right)}^{4 p}\right)^{1 / 2} \leq C(T) .
$$

Taking into account again estimate (3.1), and the uniform bound (3.2), we can infer that

$$
\mathbb{E}|I(t, x, \delta)|^{p} \lesssim_{T} \delta^{2 p} .
$$

Plugging this estimate into (5.11), we obtain that

$$
\mathbb{P}\left(\|D u(t, x)\|_{H}^{2}<\varepsilon\right) \lesssim_{T}\left(\frac{g(x, \delta)}{2}-\varepsilon\right)^{-p} \delta^{2 p} .
$$


On the other hand, (5.1) yields $g(x, \delta) \geq c_{x} \delta^{\gamma}$. Thus, if we choose $\delta=\delta(\varepsilon, x)$ sufficiently small in such a way that $c_{x} \delta^{\gamma}=4 \varepsilon$, we get

$$
\mathbb{P}\left(\|D u(t, x)\|_{H}^{2}<\varepsilon\right) \lesssim_{T} \frac{\delta^{2 p}}{\varepsilon^{p}} \lesssim_{x, T} \varepsilon^{p\left(\frac{2}{\gamma}-1\right)},
$$

where $\frac{2}{\gamma}-1>0$ by hypothesis. Therefore, going back to (5.10), it suffices to take $p=\frac{q \gamma}{2-\gamma}$ and the proof is completed.

Remark 5.9. We should point out that, in fact, in order to prove the existence of density (i.e. Theorem [5.1(a)), condition (5.1) may be slightly weakened. Precisely, one needs to prove that $\|D u(t, x)\|_{H}>0 \mathbb{P}$-a.s. First, by (5.6) and the lower semicontinuity of the norm with respect to weak convergence, one gets that for any $\delta \in(0,1)$ and $q \geq 1$,

$$
\mathbb{E}\|D u(t, x)\|_{L^{2}\left([t-\delta, t] ; L_{Q}^{2}\right)}^{q} \leq g(x, \delta)^{\frac{q}{2}} .
$$

Then, similarly as above (see also [19, Thm. 5.2]), one proves that

$$
\mathbb{E}|I(t, x, \delta)| \lesssim \delta \int_{0}^{\delta} \int_{\mathcal{O}} G_{s}(x, y) g(y, \delta) d y d s,
$$

and that, for any $n \geq 1$ and $\delta \in(0,1)$,

$$
\mathbb{P}\left(\|D u(t, x)\|_{H}^{2}<\frac{1}{n}\right) \leq\left(\frac{g(x, \delta)}{2}-\frac{1}{n}\right)^{-1} \mathbb{E}|I(t, x, \delta)| .
$$

Taking limit as $n \rightarrow \infty$ and using (5.13), we end up with

$$
\mathbb{P}\left(\|D u(t, x)\|_{H}^{2}=0\right) \lesssim g(x, \delta)^{-1} \delta \int_{0}^{\delta} \int_{\mathcal{O}} G_{s}(x, y) g(y, \delta) d y d s .
$$

In conclusion, $u(t, x)$ has a density provided that the following two conditions are satisfied:

(a) For any $x \in \mathcal{O}, g(x, \delta)>0$ for all $\delta>0$,

(b) For any $x \in \mathcal{O}$, it holds

$$
\lim _{\delta \rightarrow 0} \frac{\delta}{g(x, \delta)} \int_{0}^{\delta} \int_{\mathcal{O}} G_{s}(x, y) g(y, \delta) d y d s=0 .
$$

5.3. Proof of Theorem [5.1. It is now just a matter of putting pieces together. In particular, in view of Bouleau-Hirsch criterion (see e.g. [17, Thm. 2.1.3]), (a) follows by Propositions 5.5 and 5.8. Similarly, (b) follows by Propositions 5.7 and 5.8, applying a general criterion of the Malliavin calculus (see e.g. [17, Prop. 2.1.5] or [13, Thm. 4.1]).

\section{ACKNOWLEDGMENTS}

Part of the work for this paper was carried out while the authors were visiting the Hausdorff Institute for Mathematics in Bonn, whose hospitality and financial support are gratefully acknowledged. L. Quer-Sardanyons is also supported by the grant MICINNFEDER Ref. MTM2009-08869. 


\section{REFERENCES}

[1] V. Bally and É. Pardoux, Malliavin calculus for white noise driven parabolic SPDEs., Potential Analysis 9 (1998), no. 1, 27-64. MR 1644120 (99g:60095)

[2] H. Brézis, Opérateurs maximaux monotones et semi-groupes de contractions dans les espaces de Hilbert, North-Holland Publishing Co., Amsterdam, 1973. MR 0348562 (50 \#1060)

[3] C. Cardon-Weber, Cahn-Hilliard stochastic equation: existence of the solution and of its density, Bernoulli 7 (2001), 777-816. MR 2054575 (2005f:60136)

[4] S. Cerrai, Second order PDE's in finite and infinite dimension, Lecture Notes in Mathematics, vol. 1762, Springer-Verlag, Berlin, 2001. MR 2002j:35327

[5] G. Da Prato, Kolmogorov equations for stochastic PDEs, Birkhäuser Verlag, Basel, 2004. MR 2111320 (2005m:60002)

[6] G. Da Prato and J. Zabczyk, Stochastic equations in infinite dimensions, Cambridge University Press, Cambridge, 1992. MR 1207136 (95g:60073)

[7] R. Dalang and L. Quer-Sardanyons, Stochastic integrals for spde's: a comparison, Expo. Math. 29 (2011), 67-109. MR 2785545

[8] N. Fournier and J. Printems, Absolute continuity for some one-dimensional processes, Bernoulli 16 (2010), no. 2, 343-360. MR 2668905 (2011d:60176)

[9] H. W. Gould, The generalized chain rule of differentiation with historical notes, Util. Math. 61 (2002), 97-106. MR 1899320 (2003a:26007)

[10] I. Gyöngy and É. Pardoux, On the regularization effect of space-time white noise on quasi-linear parabolic partial differential equations, Probab. Theory Related Fields 97 (1993), no. 1-2, 211-229. MR 1240724 (94j:60123)

[11] L. V. Kantorovich and G. P. Akilov, Functional analysis, second ed., Pergamon Press, Oxford, 1982, Translated from the Russian by Howard L. Silcock. MR 664597 (83h:46002)

[12] J. A. León, D. Nualart, and R. Pettersson, The stochastic Burgers equation: finite moments and smoothness of the density, Infin. Dimens. Anal. Quantum Probab. Relat. Top. 3 (2000), no. 3, 363-385. MR 1811248 (2001m:60146)

[13] P. Malliavin, Stochastic analysis, Grundlehren der Mathematischen Wissenschaften [Fundamental Principles of Mathematical Sciences], vol. 313, Springer-Verlag, Berlin, 1997. MR 1450093 (99b:60073)

[14] D. Márquez-Carreras, M. Mellouk, and M. Sarrà, On stochastic partial differential equations with spatially correlated noise: smoothness of the law, Stochastic Process. Appl. 93 (2001), no. 2, 269284.

[15] M. Métivier and J. Pellaumail, Stochastic integration, Academic Press, New York, 1980. MR 578177 (82b:60060)

[16] A. Millet and M. Sanz-Solé, A stochastic wave equation in two space dimension: smoothness of the law, Ann. Probab. 27 (1999), no. 2, 803-844. MR 1698971 (2001e:60130)

[17] D. Nualart, The Malliavin calculus and related topics, second ed., Springer-Verlag, Berlin, 2006. MR 2200233 (2006j:60004)

[18] _ Application of Malliavin calculus to stochastic partial differential equations, A minicourse on stochastic partial differential equations, Lecture Notes in Math., vol. 1962, Springer, Berlin, 2009, pp. 73-109. MR 2508774 (2010g:60156)

[19] D. Nualart and L. Quer-Sardanyons, Existence and smoothness of the density for spatially homogeneous SPDEs, Potential Anal. 27 (2007), no. 3, 281-299. MR 2336301 (2009i:60107)

[20] É. Pardoux and Tu Sheng Zhang, Absolute continuity of the law of the solution of a parabolic SPDE, J. Funct. Anal. 112 (1993), no. 2, 447-458. MR 1213146 (94k:60095)

[21] M. Sanz-Solé, Malliavin calculus, Fundamental Sciences, EPFL Press, Lausanne, 2005, With applications to stochastic partial differential equations. MR 2167213 (2006h:60005)

[22] J. B. Walsh, An introduction to stochastic partial differential equations, École d'été de probabilités de Saint-Flour, XIV—1984, Lecture Notes in Math., vol. 1180, Springer, Berlin, 1986, pp. 265-439. MR 0876085 (88a:60114)

[23] N. L. Zaidi and D. Nualart, Burgers equation driven by a space-time white noise: absolute continuity of the solution, Stochastics Stochastics Rep. 66 (1999), no. 3-4, 273-292. MR 1692868 (2000b:60160) 
(Carlo Marinelli) Institut für Angewandte Mathematik, Universität Bonn, Germany, And Facoltà di Economia, Libera Università di Bolzano, Italy

$U R L:$ http://www . uni-bonn.de/ $\sim \operatorname{cm} 788$

(Eulalia Nualart) Institut Galilée, Université Paris 13, 93430 Villetaneuse, France

E-mail address: eulalia@nualart.es

$U R L:$ http://nualart.es

(Lluís Quer-Sardanyons) Departament de Matemàtiques, Universitat Autònoma de Barcelona, 08193 Bellaterra (Barcelona), Spain. Tel. 0034935814542, FAX 0034935812790.

E-mail address: quer@mat.uab.cat

$U R L:$ http://mat.uab.cat/ quer 